\title{
Engagement of the PFC in consolidation and recall of recent spatial memory
}

\author{
Wanda C. Leon, ${ }^{1}$ Martin A. Bruno, ${ }^{1,5}$ Simon Allard, ${ }^{1}$ Karim Nader, ${ }^{2}$ and \\ A. Claudio Cuello ${ }^{1,3,4,6}$ \\ ${ }^{1}$ Department of Pharmacology and Therapeutics, McGill University, Montreal, Quebec H3G 1Y6, Canada; ${ }^{2}$ Department of \\ Psychology, McGill University, Montreal, Quebec H3G 1Y6, Canada; ${ }^{3}$ Department of Anatomy and Cell Biology, McGill University, \\ Montreal, Quebec H3G 1Y6, Canada; ${ }^{4}$ Department of Neurology and Neurosurgery, McGill University, Montreal, Quebec H3G 1Y6, \\ Canada; ${ }^{5}$ Facultad deCiencias Médicas, UCCuyo-CONICET, San Juan J5400, Argentina
}

\begin{abstract}
The standard model of system consolidation proposes that memories are initially hippocampus dependent and become hippocampus independent over time. Previous studies have demonstrated the involvement of the medial prefrontal cortex ( $\mathrm{mPFC}$ ) in the retrieval of remote memories. The transformations required to make a memory undergo system's consolidation are thought to require synaptic plasticity. In this study, we investigated the participation of the mitogen-activated protein kinase (MAPK)/ERK pathway in acquisition, memory consolidation, and recent memory recall of the Morris water maze (MWM) task using a 1-d training protocol. To this end, bilateral injections of the MEK inhibitor U0126 into the rat $\mathrm{mPFC}$ were performed. The injection of the MEK inhibitor in the MPFC did not affect the acquisition of the MWM. However, MEK inhibitor resulted in impairments on recent memory retrieval either when applied at the end of the learning phase (memory consolidation) or prior to the retention test. The results strongly support the concept that recently acquired and consolidated spatial memories require the $\mathrm{MPFC}$, and that local activation of the MAPK/ERK pathway in the mPFC is necessary for the consolidation and recall of recent memories.
\end{abstract}

[Supplemental material is available online at http://www.learnmem.org.]

The standard model of memory system consolidation postulates that hippocampus-dependent memories become independent of the hippocampus and are stored in the neocortex over time (Marr 1971; McClelland et al. 1995; Squire and Alvarez 1995; Wiltgen et al. 2004; Frankland and Bontempi 2005; Smith and Squire 2009). In humans, damage to the hippocampus results in a disruption of recently acquired memories, while sparing remote memories (Scoville and Milner 1957). In support of such an idea, functional imaging studies from rodents and humans have indicated that hippocampal activity is associated with the retrieval of recent but not remote memories. Moreover, dorsal hippocampus inactivation impairs performance of recent, but not old memories (Maviel et al. 2004). It should be noted, however, that the temporal gradient identifying system consolidation has not usually been found (Ryan et al. 2001; Lehmann et al. 2007).

It has been proposed that the $\mathrm{mPFC}$, specifically the anterior cingulate cortex (ACC), controls the retrieval of old memories (Frankland and Bontempi 2005; Blum et al. 2006; Frankland et al. 2006). Furthermore, it has been shown that inactivation or lesion of the ACC blocks the expression of old contextual fear (Frankland et al. 2004) and spatial memories (Teixeira et al. 2006). At the synaptic level, synaptic plasticity mechanisms are thought to be required for these time-dependent changes to occur (Frankland et al. 2001; Frankland and Bontempi 2005). Prevalent theories of how hippocampus-dependent memories change over time posit that the mPFC should not undergo synaptic plasticity or be functionally necessary for the expression of recent memories (Frankland and Bontempi 2005).

\section{${ }^{6}$ Corresponding author.}

E-mail claudio.cuello@mcgill.ca; fax (514) 398-8317.

Article is online at http://www.learnmem.org/cgi/doi/10.1101//m.1804410.
Recent reports have revealed inconsistencies regarding the role of the mPFC in system's consolidation, as they have indicated that the $\mathrm{mPFC}$ is also necessary for the formation of recent memories (Zhao et al. 2005; Blum et al. 2006). Specifically, it has been demonstrated that inhibition of the NR2B subunit within the mPFC disrupts the expression of newly acquired trace-conditioned memories (Zhao et al. 2005). These findings suggest that the mPFC plays an early role in memory formation.

In this study we used a modified version of the MWM to allow the monitoring of the mitogen-activated protein kinase (MAPK)/ ERK signaling pathway by blocking and determining the degree of ERK $1 / 2$ phosphorylation as learning and memory tasks were assessed. The hidden platform version of the MWM has been used extensively to measure cognitive deficits in rodents (Morris 1984; Brandeis et al. 1989; Nieto-Escamez et al. 2004). Most of the previous work was done using a typical 5-d protocol, where the synaptic consolidation process may occur repeatedly over the training period. In our study, we used a modified single-day learning MWM protocol to better differentiate between acquisition, consolidation, and retrieval processes and for a more accurate determination of MAPK/ERK activity or its blockade. Given that extracellular signal regulated kinase-1 and 2 (ERK1/2) signaling is involved in long-term potentiation (English and Sweatt 1996, 1997; Thomas and Huganir 2004) and in learning and memory formation (Atkins et al. 1998; Blum et al. 1999; Sweatt 2001; Adams and Sweatt 2002; Silva 2003; Peng et al. 2010), we examined the expression of ERK $1 / 2$ in the hippocampus and mPFC.

To clarify whether the mPFC plays a role during recent spatial memory formation, we measured the levels of ERK activation and we transiently and selectively inhibited the ERK signaling pathway using the upstream MEK inhibitor U0126. The inhibitor was used either prior to acquisition of the MWM, right after acquisition, or after $24 \mathrm{~h}$, but 30-40 min prior to the probe test. 
We found that ERK activation was not induced in the mPFC during acquisition, but did increase during the subsequent synaptic consolidation period and during the retrieval of a new memory. However, inhibition of the ERK pathway in the mPFC resulted in an impairment of consolidation and recall of recent spatial memories without affecting the acquisition of the MWM task. These findings indicate that the acquired information is consolidated in the MPFC and that this brain region is necessary for the retrieval of recent spatial memories.

\section{Results}

Acquisition of a spatial memory induces ERK activation in the hippocampus, but not in the prefrontal cortex (PFC)

Consistent with previous studies demonstrating a role of the hippocampus in the acquisition and consolidation of recent spatial memories, ERK-phosphorylation was significantly increased in the hippocampus, but not in the PFC of rats during acquisition. Figure 1A illustrates the acquisition phase (left) and retention test (right) of rats trained on the hidden platform version of the single-day learning of the MWM. Animals required progressively less time to find the submerged platform from trials 1 to 6 , as they learned the spatial task $\left({ }^{* * *}, P<\right.$ 0.001, one-way ANOVA). Retention trials (probe tests) were performed $24 \mathrm{~h}$ after finishing the acquisition phase in the presence of the hidden platform. The latency to find the submerged platform was considered as an index of memory retention. Since our initial investigation, we have observed no significant changes in ERK phosphorylation between naïve and control rats exposed to swimming (see Supplemental Fig. S2). We performed all experiments with animals exposed to the swimming for equivalent times. Trained animals spent significantly less time searching for the platform $7.33 \pm 0.87$ (mean \pm SEM, $n=9$ ) than control animals $48.4 \pm 5.3$ (mean \pm SEM, $n=6$ ) (**, $P<0.01$, unpaired $t$-test), indicating that trained animals were able to retrieve the newly acquired spatial information $24 \mathrm{~h}$ later. The levels of hippocampal and mPFC ERK phosphorylation over acquisition trials are illustrated in Figure 1, B and C, respectively. The hippocampal ERK phosphorylation reached the maximum during the third trial $(* *, P<0.01$, one-way ANOVA), while in the mPFC no significant change was observed during the diverse stages of the training period $(P>0.05$, one-way ANOVA).

\section{ERK activation occurs in the mPFC and hippocampus during recent memory recall}

The hypothesis of systems consolidation proposes that the hippocampus, but not $\mathrm{MPFC}$, should be activated and required for the
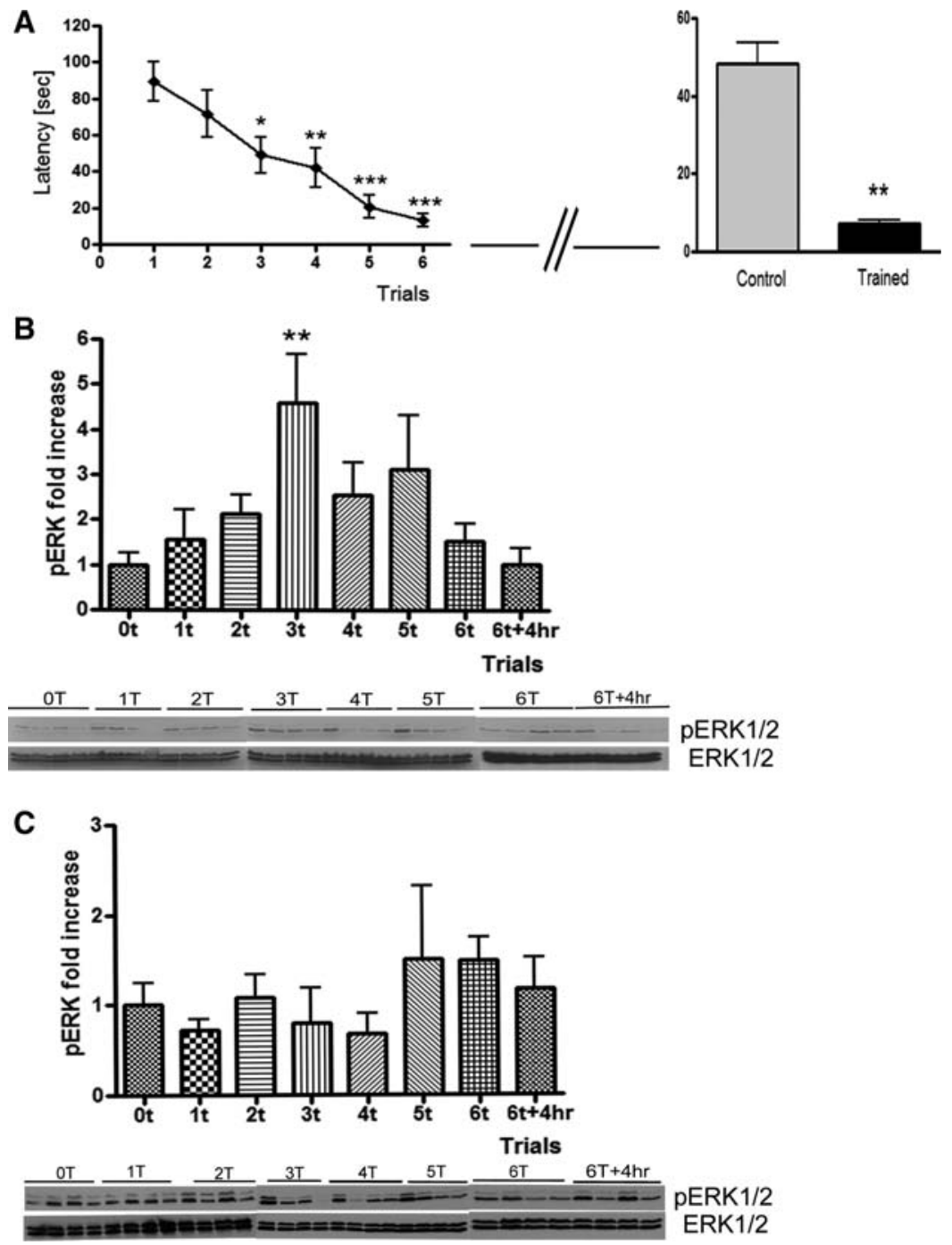

Figure 1. Acquisition of the Morris water maze increases ERK phosphorylation in the hippocampus ut not in the PFC. $(A)$ Performance of rats using the MWM task. Mean latency $\pm \operatorname{SEM}(n=9)$ to (he submerged platform (left). Note shorter latencies to find the submerged platform (seconds) from trials 1 to 6 , as rats learn the task. (Right) The retention test in the presence of hidden 列 $24 \mathrm{~h}$. As expected, trained rats $(n=9)$ found the platform significantly faster than conols $(n=6)$ (**, $P<0.01)$. $(B, C)$ Representative Western blot analysis revealing phosphorylated extracellular regulated kinase (ERK) and total ERK in hippocampus $(B)$ and in $\mathrm{MPFC}(C)$, respectively, during the training trials of MWM. Note that hippocampal ERK phosphorylation reached a peak at the third trial and returned to basal levels $4 \mathrm{~h}$ after the last trial.

expression of a hippocampus-dependent memory (Bontempi et al. 1999; Frankland et al. 2004). To investigate this issue, trained and control animals were sacrificed after the probe test, and the possible engagement of the mPFC was evaluated by measuring ERK phosphorylation levels using neurochemical and immunohistochemical methods. By quantitative Western blotting and confocal microscopy, we found that ERK was phosphorylated in both the MPFC and hippocampus after the retrieval of a 1-d-old memory. Figure 2, A and B, illustrate representative Western blots for phospho and total ERK levels during recent memory recall in both the hippocampus and mPFC of trained animals. In trained animals, we observed a significant post-training increase in ERK phosphorylation in both hippocampus $(* *, P<0.01$, unpaired $t$-test) and $\mathrm{mPFC}(*, P<0.05$, unpaired $t$-test) immediately after the retrieval of recently acquired spatial information. As shown in Figure 2, A and B, the increase in ERK phosphorylation was not a result of an increase in total ERK protein expression. To 
A
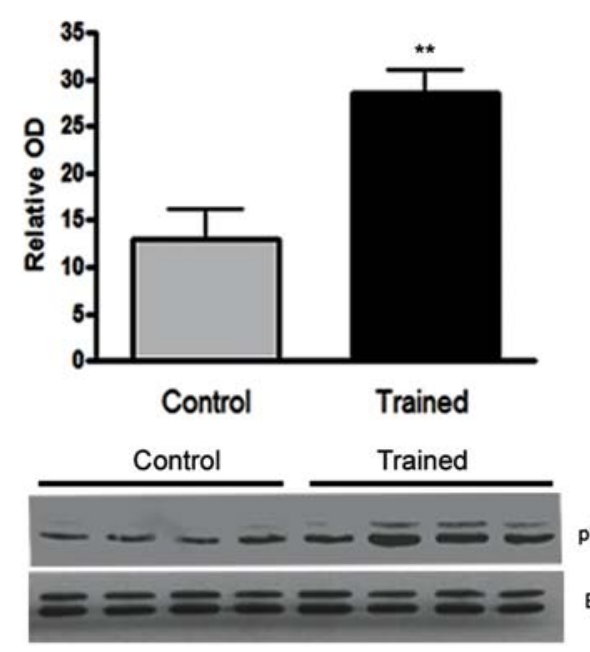

B

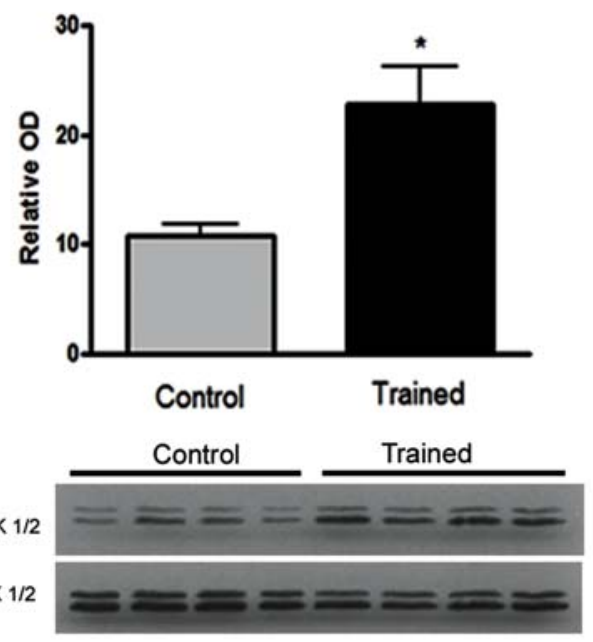

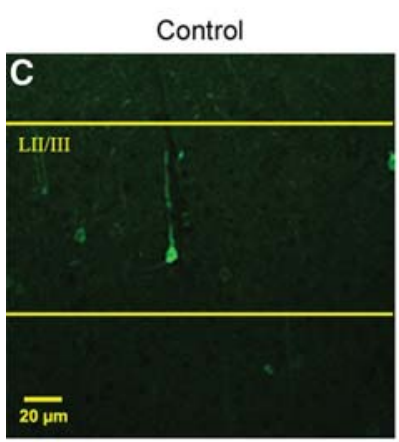

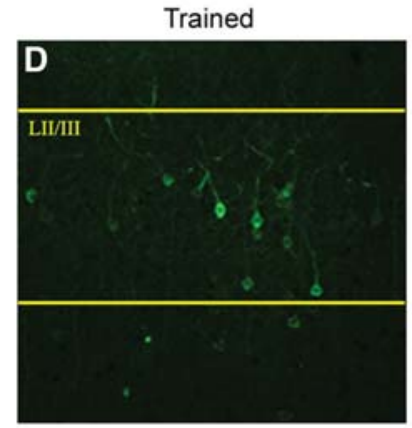

E
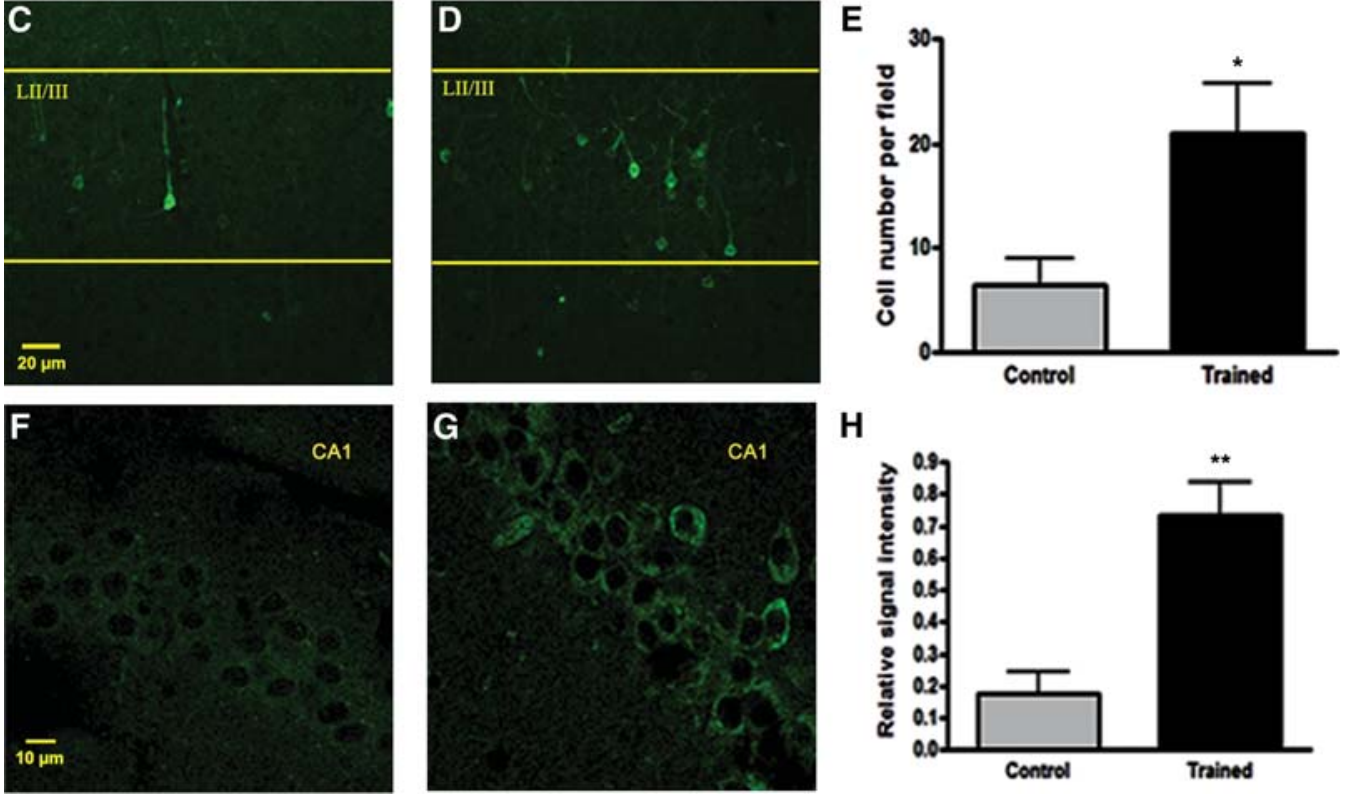

H

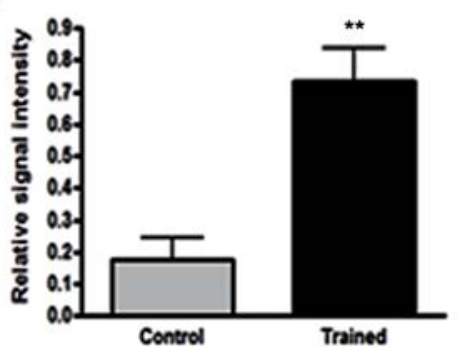

Figure 2. Stimulation of ERK pathway in both the PFC and hippocampus after 24-h retrieval of a recent spatial memory. $(A, B)$ Representative Western blot analysis revealing phosphorylated extracellular regulated kinase (pERK) and total ERK in the hippocampus and PFC. Note that ERK activation (phosphorylation) occurs during recent memory recall in both hippocampus and PFC of trained animals $(n=9)$, as revealed by the relative optical density of immunoreactions as compared with controls $(n=6)$. Statistical difference of ERK activation in the hippocampus and PFC were of $(*) P<0.05$ and $\left(*^{* *}\right) P<$ 0.01 , respectively, in relation to control groups. $(C, D)$ Phospho-ERK immunoreactivity as revealed by confocal microscopy of the PFC of the control $(n=5)$ and trained $(n=5)$ animals. The pERK immunoreactive cells were mainly present in laminae II and laminae III of the PFC. (E) Trained animals showed a significant increase in the number of immunoreactive cells when compared with control animals $(*, P<0.05)$. $(F, G)$ Representative confocal images of the CA1 region of the dorsal hippocampus of controls and trained animals, respectively, after a 24-h probe test. $(H)$ Quantification of the signal intensity in the CA1 region of control $(n=5)$ and trained animals $(n=5)$ applying an image-analysis system. The area representing the pyramidal cells was isolated using a minimum intensity threshold mask. The average brightness of the signal was then measured using the "brightness" option of the MCID software (see Materials and Methods section). The signaling intensity was significantly increased in trained animals when compared with control animals $(* *, P<0.01)$.

gather more direct information on the neuroanatomical sites of the increased ERK phosphorylation in the hippocampus and mPFC, we performed immunofluorescence and imaged by confocal microscopy (see Fig. 2C,D,F,G). Consistent with our Western blot results, there was a significant increase in the number of pERK-immunoreactive neurons observed in the MPFC of trained animals after recent memory recall $\left(^{*}, P<0.05\right.$, unpaired $t$-test; Fig. 2E). We also quantified the number of immunoreactive neurons in the FR2 region of the frontal cortex, where we did not detect significant changes after the probe test.
Furthermore, the application of the inhibitor U0126 in mPFC had no effect in basal levels of neurons displaying ERK phosphorylation in the FR2, indicating that inhibition was restricted to mPFC without affecting neighboring cortical regions (see Supplemental Fig. 3). This suggests that the phosphorylation is region specific and does not occur throughout the neocortex. In the hippocampus, we found that the average intensity of the pERK immunoreactivity was increased in the CA1 region of trained animals when compared with controls $\left(^{* *}, P<0.01\right.$, unpaired $t$-test; Fig. 2H). 
A

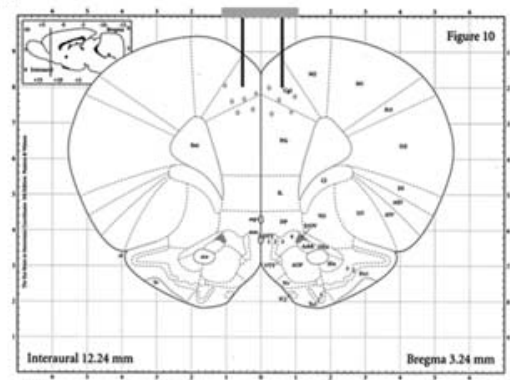

C
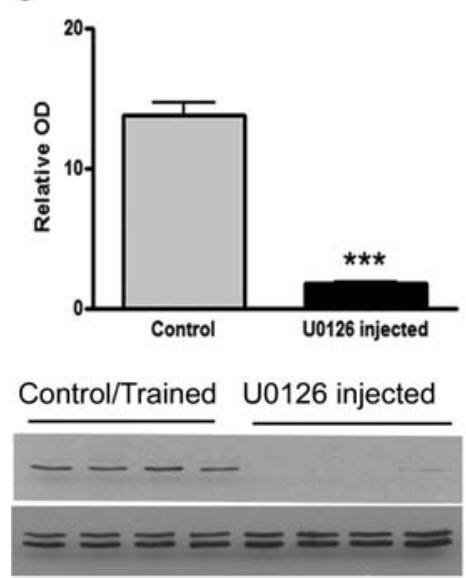

B
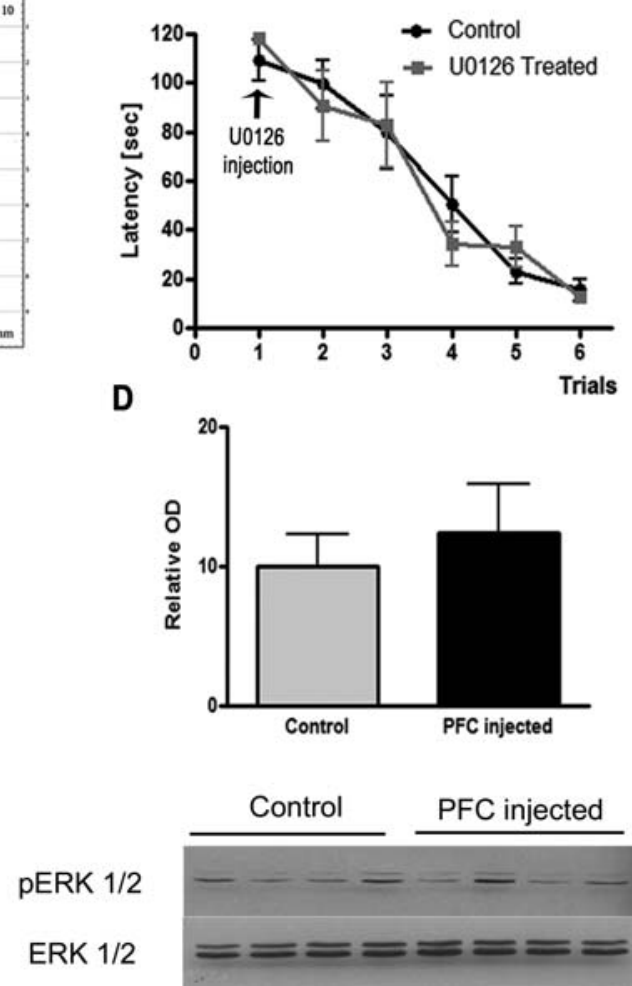

Figure 3. Learning of the MWM task is not affected by the PFC inhibition of the ERK pathway. $(A) A$ schematic representation of the cannula implantation site and drug infusion in the PFC. (B) Mean escape latency \pm SEM $(n=10)$ of rats injected with the MEK inhibitor (U0126) bilaterally in the PFC as compared with vehicle-injected $(n=10)$ rats. Note that representative Western blots show that near total inhibition of the ERK phosphorylation ( $p E R K$ ) in the PFC (C) with U0126 did not modify the learning behavior of the MWM task $(B)$. (D) The levels of phosphorylated ERK in the hippocampus were not affected by the administration in the MPFC of the U0126 MEK inhibitor. $\left(^{* * *}\right) P<0.001$.

Inhibition of ERK pathway in the mPFC did not disrupt the acquisition phase of Morris water maze task

We further investigated whether the mPFC is engaged during the acquisition phase of the single-day learning of the Morris water maze task. For this we performed a bilateral intra-mPFC infusion (Fig. 3A) of the specific MEK kinase inhibitor U0126 prior to the training. U0126 had no effect on the acquisition of the MWM task. A two-way ANOVA revealed a significant training effect $(P<0.001)$ and a nonsignificant $(P>0.05)$ training $\times$ treatment interaction (Fig. 3B). To verify independently whether U0126 injections inhibited the ERK phosphorylation pathway in the PFC area, we measured the levels of phospho ERK and total ERK by Western blotting at the end of the acquisition phase. We observed that U0126 infusion into the mPFC resulted in a significant inhibition of ERK phosphorylation $(* * *, P<0.001$ unpaired $t$-test) as compared with the vehicle-infused group (Fig. 3C). No significant ERK phosphorylation changes $(P>0.05$ unpaired $t$-test) were found in the hippocampus of rats receiving either U0126 or saline (control group) in the mPFC area (Fig. 3D).

Inhibition of the ERK pathway in the mPFC area impairs consolidation of a newly acquired spatial memory

To examine whether ERK phosphorylation in the mPFC is engaged in spatial memory during the consolidation phase, U0126 or saline was bilaterally infused after the end of the last trial of acquisition phase in single-day MWM protocol. Animals were then tested $24 \mathrm{~h}$ later for retention of memory. The mPFC-U0126 injected group spent a longer time to find the submerged platform $48.30 \pm 7.351 n=5$ (mean \pm SEM) compared with the vehicle-treated controls $5.800 \pm 0.5017 n=5(* *, P<0.01$, unpaired $t$-test; Fig. 4B). This data suggests that the mPFC plays a significant role in the synaptic stabilization of a newly acquired spatial memory.

The U0126-treated animals revealed a significant decrease in ERK phosphorylation when compared with the vehicletreated controls. No change in the total ERK protein expression was observed in any experimental group (Fig. 4C,D). The effect observed $24 \mathrm{~h}$ after the U0126 injection in Figure 4, B-D is not due to the remaining inhibitory activity of the compound. The inhibitory effects of U0126 at the injected concentration $(1.0 \mathrm{ug} / \mathrm{uL})$ in the mPFC area were transient, as no reduction in ERK phosphorylation was observed after the probe test when the animals were treated the previous day (data not shown).

\section{Inhibition of the ERK signaling} pathway in the PFC prevents the expression of recent spatial memory We further examined the role of the ERK activation in the MPFC in the recall of recent spatial memory. For this purpose, we infused U0126 in the MPFC $24 \mathrm{~h}$ after the end of the acquisition/consolidation phase and $30-40 \mathrm{~min}$ before the probe test (Fig. 5A). Animals injected with the ERK inhibitor U0126 were impaired in the expression of the acquired spatial memory. The latency to find the hidden platform was significantly higher in the U0126 treated group $(44.35 \pm 9.1 n=5)$ than in the saline group, compared with $15.08 \pm 4.8 n=5\left(^{*}, P<0.05\right.$ unpaired t-test; Fig. 5B). Rats receiving the U0126 MEK inhibitor before the probe test showed a significant inhibition of phospho ERK in the MPFC without change in the levels of total ERK as shown in Figure 5, C and D. Taken together, these results provide evidence for a direct engagement of the MPFC in both the consolidation and the recall of recently acquired spatial memory.

\section{Discussion}

Previous studies have demonstrated the loss of recent memory after bilateral surgical lesions of the hippocampus (Scoville and Milner 2000). On the other hand, system consolidation requires a time-dependent hippocampal-cortical dialogue, whereas the $\mathrm{mPFC}$ plays a critical role for storage and retrieval of remote spatial memories (Maviel et al. 2004). Moreover, Restivo et al. (2009) reported that early hippocampal lesions abolished late development of dendritic spines growth in ACC neurons in the conditioned mice, disrupting remote memory formation. However, the time course of hippocampus-PFC involvement in the storage and retrieval of recent spatial memories has not been resolved. In the present study, we report a novel finding that is relevant to the 

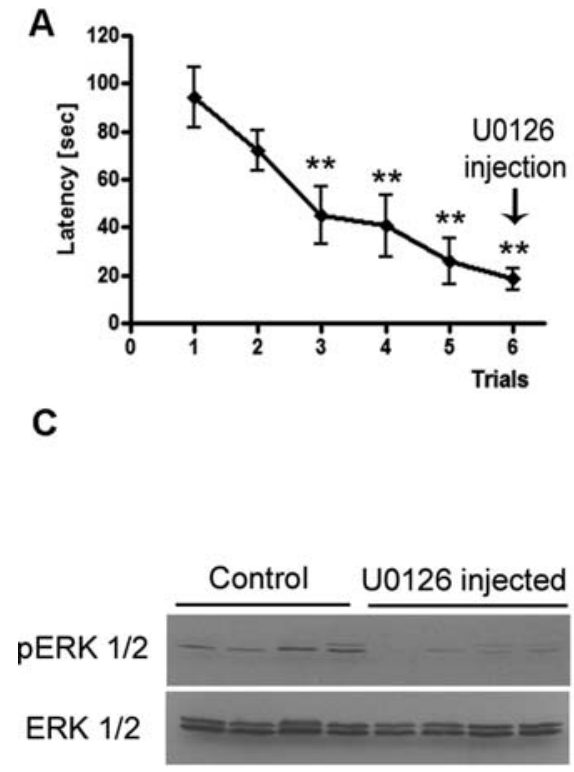
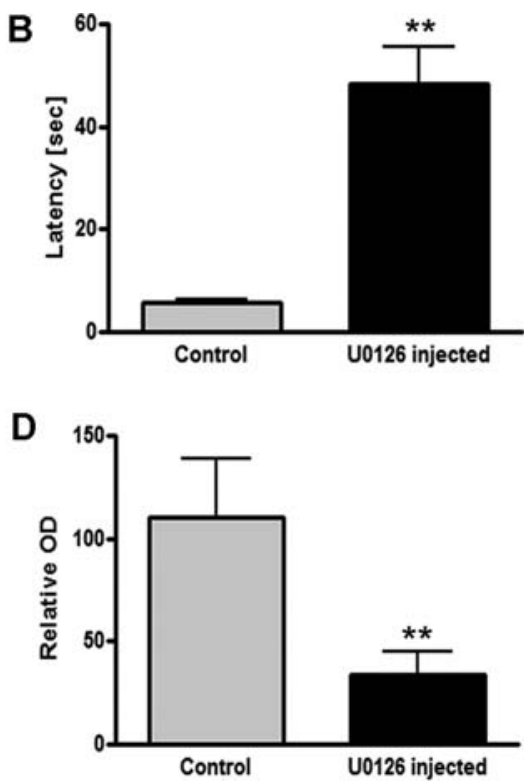

Figure 4. Post-training administration of MEK inhibitor (U0126) disrupts consolidation of recent spatial memory. $(A)$ Mean latency $\pm \operatorname{SEM}(n=10)$ to locate the submerged platform during training trials in rats injected with either vehicle $(n=5)$ or MEK inhibitor (U0126) $(n=5)(1 \mathrm{ug} / \mathrm{side})$ immediately after training. The arrow indicates initiation of treatment after learning and before retesting for "recent memory." (B) After $24 \mathrm{~h}$, the rats injected with the MEK inhibitor U0126 in the PFC fail to remember the previously learned location of the hidden platform, as revealed by the markedly increased escape latency time $(* *, P<0.01)$. (C) The effective inhibition of the activation (phosphorylation) of ERK in the PFC of treated rats, without change in total ERK protein expression, as shown in the representative Western blot and demonstrated quantitatively by analysis of the relative ( $p E R K$ normalized against total ERK) optical density of immunoreactive bands $(D)$ (**, $P<0.01)$.

involvement of the mPFC in the consolidation and retrieval of recent spatial memories. Neurochemical aspects underlying these processes were also investigated. It is difficult to separate acquisition and consolidation in the standard version of the water maze task that requires training over multiple days, where consolidation may occur repeatedly at the end of each training period. Additionally, a previous lesion study has shown that mPFC was not required for the acquisition of the classical MWM (de Bruin et al. 1994). However, when the task demand is changed by altering the configuration of the environment, the use of multiple distal cues, or by varying the number of trials, an engagement of mPFC was revealed (Compton et al. 1997). In the present study, by applying 1-d-six trials protocol for the acquisition of the MWM task, thus increasing the task demands of the MWM, we can better discriminate the acquisition from the consolidation and memory retrieval phases. This, in turn, allows us to investigate the early cell-signaling responses during these phases. We examined the ERK-cell signaling pathway, a key biochemical system classically involved in establishment of synaptic plasticity (Winder et al. 1999; Kandel 2001) and learning and memory (Sweatt 2001, 2004; Adams and Sweatt 2002).

Here we demonstrate that acquisition of spatial information of the MWM (single-day learning) induces a significant ERK activation in the hippocampus, but not in the mPFC. These results support previous reports indicating that the hippocampus is needed during spatial memory formation (Blum et al. 1999; Selcher et al. 1999; Broadbent et al. 2006) and long-term potentiation (English and Sweatt 1996). Our results would indicate that ERK activation occurs in the hippocampus during spatial learning and that the neocortex (mPFC) is involved in the subsequent memory consolidation and retrieval expression processes.
There is a body of evidence supporting the idea that aging memories become more dependent on neocortical sites (Bontempi et al. 1999; Frankland et al. 2001, 2004; Maviel et al. 2004). Our data shows that the involvement of the neocortex in consolidation is an earlier event than is proposed in the standard model of system memory consolidation (Takehara et al. 2003; Maviel et al. 2004; Frankland and Bontempi 2005; Teixeira et al. 2006). Some scientists have failed to find these gradients in rodents or human imaging (Ryan et al. 2001; Lehmann et al. 2007). Interestingly, we found that such neocortical involvement could occur in earlier stages of memory formation. Thus, during recent spatial memory recall, there was an activation of the ERK pathway not only in the hippocampus, but also in the $\mathrm{mPFC}$ after applying the novel single-day learning of the MWM. These results would be in line with a recent finding reported by Blum et al. (2006). By using a different behavioral task, they demonstrated that mPFC activation was shown to be necessary not only for remote but also recent trace fear conditioning memory. Our observations indicate that retrieval of recent memory is mPFC dependent and that its activation may depend on the executed behavioral spatial task.

Since hippocampal ERK phosphorylation has been shown to be required during recent spatial learning and memory (Blum et al. 1999; Selcher et al. 1999; Clark et al. 2005) we also investigated whether there was a role for the $\mathrm{mPFC}$ during the acquisition phase of single-day learning by inhibiting the ERK pathway in this brain area. In these experiments we found that blocking the ERK signaling pathway in the mPFC did not affect hippocampal ERK phosphorylation mechanisms during acquisition. These observations reinforce the ability of this new investigative protocol to achieve selective inhibition of the ERK pathway in the mPFC. Moreover, we have provided topographic evidence on the extent of the effects of the inhibitor (Supplemental Fig. S3), showing that the inhibition was confined to MPFC as the adjacent frontal cortical region 2 (FR2) was unaffected.

This study also demonstrated that the bilateral inhibition of the ERK pathway in the mPFC before training had no effect on the acquisition of spatial information. These results would be in agreement with a previous report in which, using a different behavioral task, Runyan and colleagues showed that mPFC does not directly participate in the encoding of fear conditioning, but impaired recent memory retention (Runyan et al. 2004). Our results show a similar involvement of the same brain region in spatial memory. Taken together, the evidence suggests that $\mathrm{mPFC}$ is involved in many types of early memory formation.

Finally, we investigated whether or not the bilateral administration of U0126 in the mPFC after the acquisition phase and before the probe test was able to disrupt recent spatial memory consolidation and retrieval. Our results revealed that MPFC ERK phosphorylation during spatial memory consolidation takes place $2 \mathrm{~h}$ after the acquisition of single-day learning of the MWM (Supplemental Fig. S1). In addition, we found that U0126 infusion into the mPFC immediately after the acquisition phase 
A

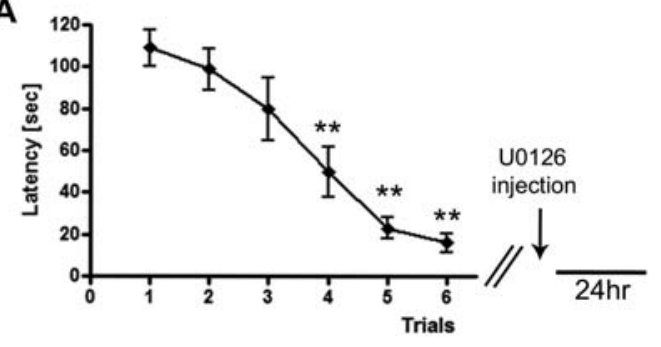

C

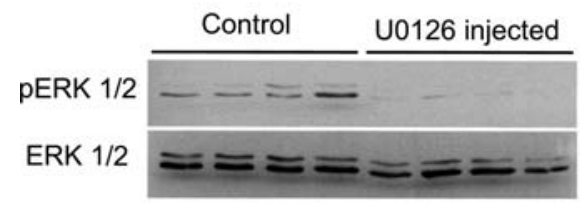

B

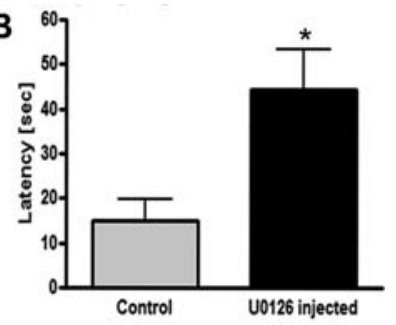

D

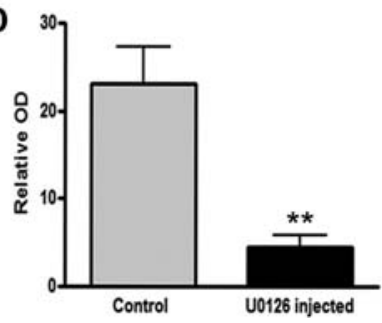

Figure 5. Disruption of the ERK pathway in the PFC after recent memory consolidation prevents memory recall. $(A)$ Performance of rats $(n=10)$ in learning the MWM task and time of injection of the MEK inhibitor in the PFC during the memory consolidation period $(* *, P<0.01)$. (B) Note the failure of PFC U0126-treated rats $(n=5)$ in retaining the acquired memory of the location of the submerged platform when the ERK pathway is disturbed after consolidation of recent memory as compared with control rats $(n=5)$. Mean escape latency $\pm \operatorname{SEM}(n=5)$ per condition ${ }^{*}, P<$ 0.05). (C) The effective drug-induced inhibition of ERK phosphorylation in the PFC by Western blot analysis. $(D)$ The effective MEK inhibitor blockade of the ERK activation as consequence of recent memory recall.

of single-day learning of the MWM blocks the synaptic consolidation of recent spatial information (Fig. 4B,C). This indicates that in our experimental paradigm the infusion of MEK inhibitor in the MPFC should have disrupted spatial memory consolidation and retrieval within the half-life of the U0126, which has been shown to be $\sim 2 \mathrm{~h}$. Therefore, behavioral testing was performed within the effective period of the compound half-life (London and Clayton 2008). These previous findings reinforce the role of $\mathrm{mPFC}$ in the consolidation and retrieval of recently acquired spatial memories. These observations are consistent with the observations of Blum et al. (2006), who demonstrated that temporary inactivation of the medial mPFC impaired recent trace fear memory recall. Other investigators have also shown that transient disconnection of the hippocampal-prefrontal circuit disrupts the retrieval of spatial memory (Floresco et al. 1997; Blum et al. 2006; Wang and Cai 2006). The above, along with the present study, support the notion that the hippocampal-prefrontal circuit does play an important role in the consolidation and retrieval of recent spatial memory.

In summary, we have shown that by using a modified version of the classical MWM (single-day learning), it is possible to manipulate the ERK cell-signaling pathway by infusing the upstream MEK inhibitor (U0126) directly and restricted to the mPFC. This approach permitted us to demonstrate that mPFC participation is necessary for the consolidation and retrieval of recent spatial memories.

\section{Materials and Methods}

\section{Animals}

Four month-old male Fischer-344 rats were used in this study, housed two to a cage with food and water ad libitum, and maintained on a 12-h light-dark cycle. All procedures were performed according to guidelines approved by the McGill University Animal Care Committee and the Canadian Council on Animal Care.

\section{Surgery}

Rats were anesthetized with equithesin and placed in a stereotaxic apparatus (David Kopf Instruments). The skin covering the skull was cut in the midline and retracted sideways. Bilateral cannulae (22-gauge stainless steel, HRS Scientific) were implanted in the mPFC (bregma $+3.2 \mathrm{~mm}$, lateral $\pm 0.75 \mathrm{~mm}$ and depth $-1.5 \mathrm{~mm}$ ) (Paxinos and Watson 1986). A solid cylindrical dummy cannula was inserted to occlude the cannula guide at the end of the surgeries. Animals were given a 7 -d rest period following surgery, before behavioral training.

\section{Pharmacological infusion}

Animals were quickly anesthetized with isoflurane (AErrane, Baxter Healthcare) in an induction chamber. The anesthesia was maintained during the U0126 infusion procedure with a table-top anesthesia system (Harvard Apparatus) equipped with a single $\mathrm{O}_{2}$ flowmeter set at $50 \mathrm{~mL} \mathrm{O} /$ minute mixed $2.5 \%$ isoflurane via a face mask.

Prior to the drug infusion each dummy guide was removed and a 28-gauge internal cannula was inserted at the depth $-1.5 \mathrm{~mm}$ prefrontal area, below the level of bregma. The cannula was connected by a polypropylene tube to a 1.0-uL Hamilton syringe that delivered the solution using an automated pump (Bioanalytical System, Inc). The selective MEK inhibitor U0126 (Promega Corp.) was dissolved in 25\% dimethyl sulfoxide (DMSO) in a phosphate-buffered saline (PBS) solution (Davies et al. 2000). A solution of $1.0 \mathrm{ug} / \mathrm{uL}$ of U0126 was infused bilaterally into PFC 30-40 min before behavioral training (pretraining), immediately after training (post-training), or $24 \mathrm{~h}$ later and before the retention probe, at a rate of $0.2 \mathrm{uL} / \mathrm{min}$ for $5 \mathrm{~min}$. The cannula was left in place for an additional 2 min to allow diffusion and to minimize backflow. At the end of infusions, the cannulae were removed and the cannula guides were occluded with the dummy cannulae. All vehicle control infusions consisted of the same volume of solution used for the pharmacological inhibitor (25\% DMSO in PBS).

\section{Behavioral procedure}

\section{Training phase}

Prior to the initiation of the behavioral procedure rats were handled during $5 \mathrm{~d}$ for a period of $5 \mathrm{~min}$ to minimize stress. Rats were trained in a modified version of the MWM task (singleday learning) (Morris et al. 1982; Morris 1984) to locate a hidden escape platform in a circular $1.4-\mathrm{m}$ pool. The pool was filled with opaque water and the testing room contained extramaze visual cues. Each rat received six trials, with an intertrial interval of $15 \mathrm{~min}$ in a single-day learning. Subjects were released into the pool from one of four starting positions, and the location of the platform remained constant throughout training (northeast quadrant of the pool). The time to find the escape platform (escape latency) was measured. If an animal failed to find the platform, it was briefly placed on the platform by the experimenter. Animals were allowed to remain on the platform for a period of $10 \mathrm{sec}$. Control animals also received six trials, allowing them to swim freely without the presence of the platform. They were allowed to swim for an average of time similar to trained animals. Visual and locomotor impairments were ruled out by raising the platform above the surface of the water. No difference was found among the experimental groups. 
For determining whether swimming in the MWM pool affected basal ERK phosphorylation levels in the MPFC, naïve (not exposed to swimming pool) $(n=5)$ and control (exposed to swimming pool) $(n=5)$ rats were sacrificed and the tissue was stored at $-80^{\circ} \mathrm{C}$ for further analysis. The Western blot analysis of ERK phosphorylation revealed no changes between naïve and control animals, and therefore consecutive studies were carried out only with control rats (see Supplemental Fig. S2).

For the recent spatial memory recall experiments, control $(n=6)$ and trained rats $(n=9)$ were trained in a single day of the MWM.

In the pretraining experiment (before acquisition of MWM) rats were given a recovery period of 30-40 min after the infusion with either $1.0 \mathrm{ug} / \mathrm{uL}$ of U0126 or saline $(n=10)$, respectively. In the post-training experiment (immediately after acquisition of MWM) rats were infused with either U0126 $(n=5)$ or saline $(n=5)$ and retested after $24 \mathrm{~h}$. For the mPFC consolidation phase experiment ( $n=5$ per time point) rats per time point were sacrificed at $0,60,120$, and $240 \mathrm{~min}$ after the acquisition of MWM task. Finally, $24 \mathrm{~h}$ after the acquisition phase of MWM and 30$40 \mathrm{~min}$ before the retention probe, rats were injected with either U0126 $(n=5)$ or saline $(n=5)$.

For the time course ERK phosphorylation experiments in the $\mathrm{mPFC}$ and hippocampus during acquisition of 1-d protocol, rats were sacrificed right after each trial $(n=4)$.

For the immunocytochemical analysis of ERK phosphorylation, $(n=5)$ trained and $(n=5)$ control animals were used. All animals were sacrificed shortly after the retention probe, $24 \mathrm{~h}$ after acquisition.

\section{Retention probe}

After completing the single-day learning of the MWM, memory recall was determined by a probe test. Recent memory formation was evaluated by the latency for each animal to find the submerged platform in one trial of $60 \mathrm{sec}$. This probe test was performed $24 \mathrm{~h}$ after the acquisition, and measured the ability to consolidate and retrieve recent spatial memories.

\section{Preparation of protein extracts}

At the appropriate time points, immediately after acquisition, consolidation and recent spatial memory recall, animals were sacrificed and the brain tissue (hippocampal and medial prefrontal area) was quickly removed. Both mPFC tissue and whole hippocampus were homogenized in $10 \times$ cell lysis buffer (Cell Signaling Technology, Inc.) and protease inhibitor cocktail (Roche Diagnostics).

\section{Western blot analysis}

Samples of whole hippocampus and mPFC homogenate from individual rats were normalized by total protein content through dilution with cell lysis buffer. The amount of protein was measured using a conventional protein assay protocol (Bio-Rad Laboratories, Inc.) using Bovine Serum Albumin (BSA) as the standard. Total homogenate (15 uL) (50-60 ug of total protein) was resolved in $12 \%$ polyacrylamide gels and transferred into a nitrocellulose membrane (Bio-Rad Laboratories, Inc). Nitrocellulose membranes were probed with two primary antibodies specific for p44/42 ERK kinase and phospho-p44/42 ERK kinase antibodies (Cell Signaling Technology, Inc.).

Membranes were blocked in Tris Buffer Solution-Tween 20 (TBS-T) containing 10\% nonfat dry milk at room temperature for $1 \mathrm{~h}$ on a slow shaker. Then, membranes were incubated overnight at $4^{\circ} \mathrm{C}$ in solutions containing the primary antibodies (1:1000). After primary antibody incubation, membranes were washed $3 \times 10 \mathrm{~min}$ in TBS-T. This was followed by incubation for $1 \mathrm{~h}$ at room temperature with a peroxidase-conjugated goat anti-rabbit Ig antibody (1:5000) (Jackson Immuno Research Laboratories, Inc.). The membranes were washed $3 \times 10 \mathrm{~min}$ in TBS-T. The immunoreactive bands were visualized with the ECL enhanced chemiluminescence kit (Amersham Bioscience) and using Kodak Biomax XAR imaging film. The immunoreactive bands were determined by densitometry of the films using MCID image analysis system. The phosphorylated forms of ERK were normalized to total ERK.

\section{Immunocytochemical analysis}

All animals were deeply anesthetized to areflexia with $0.4 \mathrm{~mL} / \mathrm{kg}$ of Equithesin, $6.5 \mathrm{mg}$ of chloral hydrate, and $3 \mathrm{mg}$ of sodium pentobarbital in a volume of $0.3 \mathrm{~mL}$, i.p. per $100 \mathrm{~g}$ body weight. Anesthetized animals were perfused transcardially with cold saline, followed by a solution of $4 \%$ paraformaldehyde in $0.1 \mathrm{M}$ phosphate puffer ( $\mathrm{PB}),(\mathrm{pH}$ 7.4). Rat brains were then removed, post-fixed for $4 \mathrm{~h}$ in the same fixative solution, and then cryoprotected in $30 \%$ sucrose in $0.1 \mathrm{M} \mathrm{PB}$.

Tissues were embedded in an optimal cutting temperature (OCT) compound (Tissue-Tek) and $35-\mu \mathrm{m}$-thick sections were cut on a cryostat (Leica CM-3050-S) and collected in PBS containing $0.2 \%$ Triton-X 100 (PBS $+\mathrm{T}$ ). Brain sections were washed for 30 min with PBS $+\mathrm{T}$ and incubated in $10 \%$ normal goat serum (NGS) for $60 \mathrm{~min}$.

The tissue was then incubated at $4^{\circ} \mathrm{C}$ for $24 \mathrm{~h}$ in $5 \% \mathrm{NGS}$ and a polyclonal antibody raised in rabbit against the phosphorylated ERK kinase (Cell Signaling Technology, Inc.) at a concentration of 1 in 50 in PBS + T. Sections were then washed for $30 \mathrm{~min}$ in PBS + $\mathrm{T}$, following which they were incubated for $2 \mathrm{~h}$ at room temperature in a goat anti-rabbit biotinylated IgG (1:200; Vector). The sections were washed again with PBS $+\mathrm{T}$ and incubated for $2 \mathrm{~h}$ at room temperature in Alexa Fluor 488 conjugated to streptavidin (1:200; Molecular Probes). Sections were then washed for 30 min in PBS and mounted on gelatin-subbed slides, dried overnight, and coverslipped with Aqua Polymount (Polysciences, Inc.). Changes in pERK immunoreactivity were determined by capturing images of immunoreactive cells using a Zeiss LSM 510 confocal microscope equipped with an argon laser. All settings regarding lasers and digital gain were kept constant for all images from all animals. Confocal images were exported as TIFF files and analyzed with an image analysis system (MCID Elite version 7, Imaging Research, Inc.). For both the PFC and the hippocampus, three pictures, corresponding to three different fields, were taken per section, and four sections per animal were used. A total of 12 pictures for the PFC and 12 pictures for the hippocampus were quantified per animal. More precisely, we quantified in the Cg1 and $\mathrm{Cg} 2$ areas of the anterior cingulate cortex and the CA1 region of the hippocampus since they were the regions with the most apparent phosphorylation.

\section{Quantification of pERK immunoreactive neurons in the $\mathrm{mPFC}$}

Immunoreactive neurons were counted using the MCID image analysis software. In short, a brightness threshold was set to separate immunoreactive cell bodies from the background. The software considered continuous areas above threshold that were bigger than $10 \mu \mathrm{m}^{2}$ as cell bodies. All of the cell bodies were added for each confocal image and these counts were averaged for each animal. To control for proper quantification, two animals were manually counted and the numbers were compared with the ones generated by the MCID software. The neuronal counts were highly comparable using both techniques. The images were always taken in the $\mathrm{Cg} 1$ and $\mathrm{Cg} 2$ areas of the mPFC.

\section{Quantification of the hippocampus}

A threshold for confocal images was obtained using a brightness criterion to isolate immunoreactive neurons. The minimal intensity threshold was kept consistent throughout all confocal images and reliably isolated immunoreactive neurons. Following thresholding, the brightness index was obtained from the MCID image analysis software that represented the average brightness level of all immunoreactive material that was above the minimal 
brightness criterion. A relative brightness of 1 means that all the selected pixels were saturated and a relative brightness index of 0 means that all of the pixels were black. Since all of the tissue was incubated with the same antibody mixtures and imaged with consistent confocal settings, we interpreted the relative brightness as an index of protein levels. Due to a different cellular organization and a different distribution of the immunoreactivity, we could not properly isolate a specific immunoreactive neuronal population in the CA1 region of the hippocampus and quantify the number of neurons as we did in the mPFC. Therefore, the relative brightness of the overall neuronal population per confocal image was quantified in an attempt to find a cytological equivalent of the data obtained by Western blotting.

\section{Statistics}

For the behavioral studies, the data was analyzed for statistical significance using a one-way ANOVA and two-way ANOVA. Comparisons between groups were done using a two-tailed unpaired $t$-test. A value of $P<0.05$ was used as a criterion for statistical significance.

\section{Acknowledgments}

This work was supported by Canadian Institutes of Health Research grant no. MOP-89360 to A.C.C. W.C.L. is the recipient of a Fellowship from the University of Los Andes, Merida-Venezuela, and A.C.C. is the holder of the McGill University Charles E. Frosst Merck Chair of Pharmacology. We thank Nouk Tanke, Florencia Iulita, and Nikki Hassell for their contribution in editing this manuscript.

\section{References}

Adams JP, Sweatt JD. 2002. Molecular psychology: Roles for the ERK MAP kinase cascade in memory. Annu Rev Pharmacol Toxicol 42: $135-163$.

Atkins CM, Selcher JC, Petraitis JJ, Trzaskos JM, Sweatt JD. 1998. The MAPK cascade is required for mammalian associative learning. Nat Neurosci 1: 602-609.

Blum S, Moore AN, Adams F, Dash PK. 1999. A mitogen-activated protein kinase cascade in the CA1/CA2 subfield of the dorsal hippocampus is essential for long-term spatial memory. J Neurosci 19: $3535-3544$

Blum S, Hebert AE, Dash PK. 2006. A role for the prefrontal cortex in recall of recent and remote memories. Neuroreport 17: 341-344.

Bontempi B, Laurent-Demir C, Destrade C, Jaffard R. 1999. Time-dependent reorganization of brain circuitry underlying long-term memory storage. Nature 400: 671-675.

Brandeis R, Brandys Y, Yehuda S. 1989. The use of the Morris Water Maze in the study of memory and learning. Int J Neurosci 48: 29-69.

Broadbent NJ, Squire LR, Clark RE. 2006. Reversible hippocampal lesions disrupt water maze performance during both recent and remote memory tests. Learn Mem 13: 187-191.

Clark RE, Broadbent NJ, Squire LR. 2005. Hippocampus and remote spatial memory in rats. Hippocampus 15: 260-272.

Compton DM, Griffith HR, McDaniel WF, Foster RA, Davis BK. 1997. The flexible use of multiple cue relationships in spatial navigation: A comparison of water maze performance following hippocampal, medial septal, prefrontal cortex, or posterior parietal cortex lesions. Neurobiol Learn Mem 68: 117-132.

Davies SP, Reddy H, Caivano M, Cohen P. 2000. Specificity and mechanism of action of some commonly used protein kinase inhibitors. Biochem 351: $95-105$.

de Bruin JP, Sanchez-Santed F, Heinsbroek RP, Donker A, Postmes P. 1994. A behavioural analysis of rats with damage to the medial prefrontal cortex using the Morris water maze: Evidence for behavioural flexibility, but not for impaired spatial navigation. Brain Res 652: $323-333$.

English JD, Sweatt JD. 1996. Activation of p42 mitogen-activated protein kinase in hippocampal long term potentiation. J Biol Chem 271: 24329-24332.

English JD, Sweatt JD. 1997. A requirement for the mitogen-activated protein kinase cascade in hippocampal long term potentiation. J Biol Chem 272: 19103-19106.
Floresco SB, Seamans JK, Phillips AG. 1997. Selective roles for hippocampal, prefrontal cortical, and ventral striatal circuits in radial-arm maze tasks with or without a delay. J Neurosci 17: 1880-1890.

Frankland PW, Bontempi B. 2005. The organization of recent and remote memories. Nat Rev Neurosci 6: 119-130.

Frankland PW, O'Brien C, Ohno M, Kirkwood A, Silva AJ. 2001. $\alpha$-CaMKII-dependent plasticity in the cortex is required for permanent memory. Nature 411: 309-313.

Frankland PW, Bontempi B, Talton LE, Kaczmarek L, Silva AJ. 2004. The involvement of the anterior cingulate cortex in remote contextual fear memory. Science 304: 881-883.

Frankland PW, Ding HK, Takahashi E, Suzuki A, Kida S, Silva AJ. 2006. Stability of recent and remote contextual fear memory. Learn Mem 13: $451-457$.

Kandel ER. 2001. The molecular biology of memory storage: A dialogue between genes and synapses. Science 294: 1030-1038.

Lehmann H, Lacanilao S, Sutherland RJ. 2007. Complete or partial hippocampal damage produces equivalent retrograde amnesia for remote contextual fear memories. Eur J Neurosci 25: 1278-1286.

London SE, Clayton DF. 2008. Functional identification of sensory mechanisms required for developmental song learning. Nat Neurosci 11: $579-586$

Marr D. 1971. Simple memory: A theory for archicortex. Philos Trans R Soc Lond B Biol Sci 262: 23-81.

Maviel T, Durkin TP, Menzaghi F, Bontempi B. 2004. Sites of neocortical reorganization critical for remote spatial memory. Science 305: $96-99$

McClelland JL, McNaughton BL, O'Reilly RC. 1995. Why there are complementary learning systems in the hippocampus and neocortex Insights from the successes and failures of connectionist models of learning and memory. Psychol Rev 102: 419-457.

Morris R. 1984. Developments of a water-maze procedure for studying spatial learning in the rat. J Neurosci Methods 11: 47-60.

Morris RG, Garrud P, Rawlins JN, O'Keefe J. 1982. Place navigation impaired in rats with hippocampal lesions. Nature 297: 681-683.

Nieto-Escamez FA, Sanchez-Santed F, de Bruin JPC. 2004. Pretraining or previous non-spatial experience improves spatial learning in the Morris water maze of nucleus basalis lesioned rats. Behav Brain Res 148: $55-71$.

Paxinos G, Watson C. 1986. The rat brain in stereotaxic coordinates. Academic Press, Sidney, Australia.

Peng S, Zhang Y, Zhang J, Wang H, Ren B. 2010. ERK in learning and memory: A review of recent research. J Mol Sci 11: 222-232.

Restivo L, Vetere G, Bontempi B, Ammassari-Teule M. 2009. The formation of recent and remote memory is associated with time-dependent formation of dendritic spines in the hippocampus and anterior cingulate cortex. J Neurosci 29: 8206-8214.

Runyan JD, Moore AN, Dash PK. 2004. A role for prefrontal cortex in memory storage for trace fear conditioning. J Neurosci 24: $1288-1295$.

Ryan L, Nadel L, Keil K, Putnam K, Schnyer D, Trouard T, Moscovitch M. 2001. Hippocampal complex and retrieval of recent and very remote autobiographical memories: Evidence from functional magnetic resonance imaging in neurologically intact people. Hippocampus 11: $707-714$.

Scoville WB, Milner B. 1957. Loss of recent memory after bilateral hippocampal lesions. J Neurol Neurosurg Psychiatry 20: 11-21.

Scoville WB, Milner B. 2000. Loss of recent memory after bilateral hippocampal lesions. 1957. J Neuropsychiatry Clin Neurosci 12: $103-113$.

Selcher JC, Atkins CM, Trzaskos JM, Paylor R, Sweatt JD. 1999. A necessity for MAP kinase activation in mammalian spatial learning. Learn Mem 6: $478-490$.

Silva AJ. 2003. Molecular and cellular cognitive studies of the role of synaptic plasticity in memory. J Neurobiol 54: $224-237$.

Smith CN, Squire LR. 2009. Medial temporal lobe activity during retrieval of semantic memory is related to the age of the memory. J Neurosci 29: 930-938.

Squire LR, Alvarez P. 1995. Retrograde amnesia and memory consolidation: A neurobiological perspective. Curr Opin Neurobiol 5: 169-177.

Sweatt JD. 2001. The neuronal MAP kinase cascade: A biochemical signal integration system subserving synaptic plasticity and memory. $J$ Neurochem 76: $1-10$.

Sweatt JD. 2004. Mitogen-activated protein kinases in synaptic plasticity and memory. Curr Opin Neurobiol 14: 311-317.

Takehara K, Kawahara S, Kirino Y. 2003. Time-dependent reorganization of the brain components underlying memory retention in trace eyeblink conditioning. I Neurosci 23: 9897-9905.

Teixeira CM, Pomedli SR, Maei HR, Kee N, Frankland PW. 2006. Involvement of the anterior cingulate cortex in the expression of remote spatial memory. J Neurosci 26: 7555-7564. 
Thomas GM, Huganir RL. 2004. MAPK cascade signalling and synaptic plasticity. Nat Rev Neurosci 5: 173-183.

Wang GW, Cai JX. 2006. Disconnection of the hippocampal-prefrontal cortical circuits impairs spatial working memory performance in rats. Behav Brain Res 175: 329-336.

Wiltgen BJ, Brown RA, Talton LE, Silva AJ. 2004. New circuits for old memories: The role of the neocortex in consolidation. Neuron 44: 101-108.

Winder DG, Martin KC, Muzzio IA, Rohrer D, Chruscinski A, Kobilka B, Kandel ER. 1999. ERK plays a regulatory role in induction of LTP by $\theta$ frequency stimulation and its modulation by $\beta$-adrenergic receptors. Neuron 24: 715-726.

Zhao MG, Toyoda H, Lee YS, Wu LJ, Ko SW, Zhang XH, Jia Y, Shum F, Xu H, Li BM, et al. 2005. Roles of NMDA NR2B subtype receptor in prefrontal long-term potentiation and contextual fear memory. Neuron 47: $859-872$.

Received March 17, 2010; accepted in revised form April 14, 2010. 


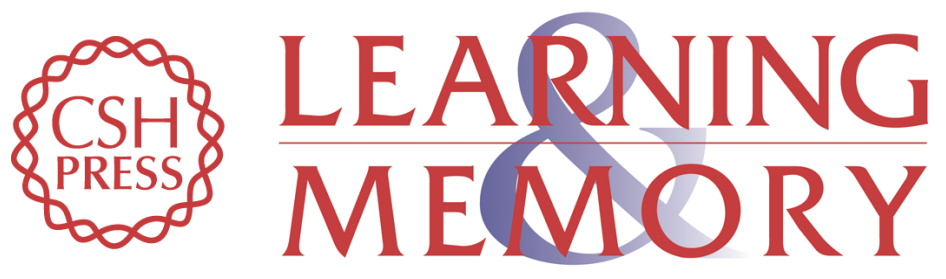

\section{Engagement of the PFC in consolidation and recall of recent spatial memory}

Wanda C. Leon, Martin A. Bruno, Simon Allard, et al.

Learn. Mem. 2010, 17:

Access the most recent version at doi:10.1101//m.1804410

Supplemental
Material http://learnmem.cshlp.org/content/suppl/2010/05/27/17.6.297.DC1

References This article cites 46 articles, 16 of which can be accessed free at: http://learnmem.cshlp.org/content/17/6/297.full.html\#ref-list-1

License

Email Alerting Receive free email alerts when new articles cite this article - sign up in the box at the Service top right corner of the article or click here. 